\title{
Effects of a Mask on Breathing Impairment During a Fencing Assault: A Case Series Study
}

\author{
Desiderio Passali,, Jacopo Cambi, ${ }^{1}$, Lorenzo Salerni, ${ }^{1}$ Giancarlo Stortini, ${ }^{1}$ Luisa Maria \\ Bellussi, ${ }^{1}$ and Francesco Maria Passali ${ }^{2}$ \\ ${ }^{1}$ ENT Department, University of Siena, Siena, Italy \\ ${ }^{2}$ ENT Department, University of Rome Tor Vergata, Rome, Italy \\ *Corresponding author: Desiderio Passali, ENT Department, University of Siena, Siena, Italy. E-mail: d.passali@virgilio.it
}

Received: August 20, 2013; Accepted: March 5, 2014

\begin{abstract}
Background: Fencers often complain of progressive difficulty in breathing during matches, which is generally attributed to restricted air, light and heat circulation from wearing a mask. Physiologically, the nasal structure generates airflow resistance that can reach - $50 \%$ of the total respiratory resistance.

Objectives: This study aims to investigate the presence of nasal obstruction in fencers and the relationship with the use of mask.

Materials and Methods: An observational study on 40 fencers (18 males, 22 females) was conducted. Fencers perform a usual assault, wearing the mask and standardized physical exercises (running, sprints and obstacles) without the mask. ENT examination with a nasal flexible fiberscope, Anterior Active Rhinomanometry (AAR) and Peak Nasal Inspiratory Flow (PNIF)measurement before and after physical activity with or without the mask was recorded.

Results: Before physical exercise, the total nasal airway resistance mean value for AAR was $0.33 \pm 0.17 \mathrm{~Pa} / \mathrm{cm}^{3} / \mathrm{s}$ at $150 \mathrm{~Pa}$. After a match with the mask, the mean value was $0.28 \pm 0.16 \mathrm{~Pa} / \mathrm{cm}^{3} / \mathrm{s}$. After normal physical exercises without mask, the mean value was $0.24 \pm 0.15 \mathrm{~Pa} / \mathrm{cm}^{3} / \mathrm{s}$. Using t tests, statistically significant difference between nasal resistance before and after physical activity $(\mathrm{P}<0.05)$ was observed, but no significant difference in nasal resistance between the basal value and that taken after a match wearing the masks $(P=0.1265)$. PNIF values significantly increase with exercise $(\mathrm{P}<0.05)$.

Conclusions: Our study shows that wearing the mask causes increased breathing impairment in fencers, when compared with similar physical activity without the mask.
\end{abstract}

Keywords: Fencing; Nasal Obstruction; Rhinomanometry; Peak Nasal Inspiratory Flow

\section{Background}

Fencers often complain of progressive difficulty in breathing during matches, which is generally attributed to restricted air, light, and heat circulation from wearing a mask. Physiologically, the nasal structure generates airflow resistance that can reach $-50 \%$ of the total respiratory resistance. The Peak Nasal Inspiratory Flow (PNIF) evaluation is the gold standard for a physiologic measure of nasal air during forced inspiration, which assesses airflow during respiration in liters per minute, as air passes through a tube, which is connected to an anesthesia mask, closed over the nose and mouth. While this method is cheap, quick and easy to use, it does not allow for unilateral measurements and evaluation in patients with alar collapse (1).

Active Anterior Rhinomanometry (AAR) permits a physiologic evaluation of nasal pressure and airflow during normal inspiration and expiration. It is considered the standard technique for a quantitative measure of nasal airflow resistance when performed according to the rules suggested by the Committee on Standardization. Normal values for total nasal resistance are $<0.25 \mathrm{~Pa} /$ $\mathrm{cm}^{3} / \mathrm{s}$ at $150 \mathrm{~Pa}(2)$. No difference in normal values of nasal resistance was observable between trained athletes and sedentary individuals (3). Aerobic exercise augments the nasal volume during an acoustic rhynometry evaluation. This increase of volume is temporary; twenty minutes after physical exercise ends, total nasal volume returns to the basal levels (4). African-Americans have a greater nasal contribution to breathing during exercise than Caucasians. Women also had a greater nasal contribution to breathing during exercise than men (5).

During exercise, nasal resistance decreases because of a combination of sympathetic nervous system mucosal vasoconstriction and alae nasi muscle recruitment, which lowers nasal vestibule resistance. In a similar fashion, external nasal dilator strips harden the lateral nasal vestibule walls, thus avoiding nasal vestibule narrowing and the development of inspiratory nasal airflow limitation (6-8). 
Passali D et al.

\section{Objectives}

The main aim of this study was to assess the presence of a nasal obstruction due only to physical activity performed with fencing mask in professional athletes. Presence of nasal predisposing factors, such as septal deviation or turbinate hypertrophy, was investigated.

\section{Materials and Methods}

Between January 2010 and January 2013, a cohort of 40 athletes (18 males and 22 females, aged from 12 to 32 years, mean age $16 \pm 3.4$ years) were analyzed for an observational study in the CUS Fencing Siena University Gym by our ENT Department. The inclusion criteria to be part of the study was to be a professional fencer at least two years, not having undergone nasal surgery, not having used nasal therapies in the last three months (steroid sprays), not being smokers. ENT general examination with nasal fiberscope endoscopy with particular attention to the nasal area was performed. Measurement of head circumference, size of mask, weapon and an estimation of the hair volume by a scale from 0 to 4 was noted. All athletes wore masks approved by Federation Internationale d'Escrime and by Federazione Italiana Scherma (Negrini, Absolute Fencing, Allstar).

For AAR, we used rhinomanometry (Atmos MedizinTechnik, GmbH and Co. KG) and for the PNIF value, we use an In-Check Oral (Clement Clark International), attached to a transparent anesthesia mask. An AAR was performed initially in basal conditions for all athletes, after they were divided into two groups. In the first group, measurements of nasal airflow were taken after a single assault, and 60 minutes later, the subjects performed a standardized course of physical activity for 15 minutes, whereupon a final measurement was taken. The second group performed the activities in reverse order. The physical activity without mask was designed to be as similar as possible to the activity that a fencer takes usually during an assault, consisting of very brief actions, fairly anaerobic. For the PNIF measurement, the nasal flow is expressed in liters per minute and five consecutive measurements are performed. The resulting measure is the mean of the two best outcomes with less than $10 \%$ variation.

The study was approved by the local Ethics Committee. Written informed consent for investigation was given by the parents if minor or directly by the athlete, and the study was conducted in accordance with the declaration of Helsinki. Comparisons between groups were assessed using paired t-test, as appropriate, at a significance level of $\mathrm{P}<0.05$. Statistical analysis was performed with SPSS software (SPSS, Inc., Chicago, IL, USA).

\section{Results}

Clinical findings during ENT valuation and distinctive characteristics of fencers were reported in Table 1. Twenty-three athletes (57.5\%) reported episodes of significant nasal obstruction during assaults. There was no correlation between size of cranium, hair volume and size of mask or weapons and AAR and PNIF values. A summary of AAR and PNIF mean values is reported in Table 2. There was a statistically significant difference using the t-test, when considering nasal resistance values (right nostril, left nostril, and total) before physical activity and after physical activity, without mask $(\mathrm{P}=0.02$ for right nostril, $\mathrm{P}=0.003$ for left nostril, $\mathrm{P}=0.003$ for total resistance) . No statistically significant differences were founded with t-tests between nasal resistance values before physical activity and after an assault with mask ( $\mathrm{P}=0.1$ for right nostril, $\mathrm{P}=0.05$ for left nostril, $\mathrm{P}=0.1$ for total resistance). There was a statistically significant difference between PNIF basal value and that collected after physical activity, with and without mask $(\mathrm{P}=0.0037$ and $\mathrm{P}=0.0006$, respectively).

\section{Discussion}

The results confirm the hypothesis deducted from the experiences of fencers. The mask creates an unfavorable environment for breathing ability, as assessed by a nasal resistances value and it negatively impacts the flow of air, which causes discomfort. When viewing the rhinomanometric results, it was easy to see a significantly greater impairment in inspiratory flow, and thus a relative increase in inspiratory resistance.

Table 1. Clinical Findings and Distinctive Characteristics of Fencers ${ }^{a}$

\begin{tabular}{|cc}
\hline Variables & Values \\
\hline ENT Valuation & \\
\hline Allergy (Congestion of nasal mucosa) & $6(15)(4)$ \\
\hline Inferior turbinate hypertrophy (A) & $9(22.5)$ \\
\hline Septal deviation (B) & $20(50)$ \\
\hline A +B & $8(20)$ \\
\hline Pharyngeal tonsil hypertrophy & $2(5)$ grade $3+$ or $4+$ \\
\hline Head Measurements & \\
\hline Cranial circumference & $56.21(2.84) \mathrm{cm}$ \\
\hline Hair volume & $($ range: $52-65)$ \\
\hline 0 & \\
\hline 1 & $16(40)$ \\
\hline 2 & $2(5)$ \\
\hline 3 & $12(30)$ \\
\hline 4 & $7(17.5)$ \\
\hline Weapon & $3(7.5)$ \\
\hline Foil & \\
\hline Epee & $30(75)$ \\
\hline Sabre & $6(15)$ \\
\hline
\end{tabular}


Table 2. Mean Value at Anterior Active Rhinomanometry (AAR) and Peak Nasal Inspiratory Flow (PNIF) Before Physical Activity and After With or Without Mask

\begin{tabular}{lccc}
\hline & Before & After Mask & After Physical Activity \\
\hline AAR, Pa/cm ${ }^{3} / \mathbf{s}$ at 150 Pa & & & \\
Right nostril, (range) & $0.73(0.60),(0.21-3.75)$ & $0.56(0.32),(0.14-1.33)$ & $0.49(0.30),(0.08-1.50)$ \\
Left nostril, (range) & $0.76(0.61),(0.23-2.88)$ & $0.58(0.30),(0.25-1.56)$ & $0.50(0.39),(0.14-1.93)$ \\
Total, (range) & $0.33(0.17),(0.11-0.81)$ & $0.28(0.16),(0.07-0.68)$ & $0.24(0.15),(0.09-0.78)$ \\
PNIF, L/min, (range) & $103.51(41.39),(40-250)$ & $126.90(41.40),(60-250)$ & $133.78(41.17),(50-250)$ \\
\hline
\end{tabular}

The uncomfortable feeling of lacking air could be caused by the athletic competition, the type of attack, psychological messages from the adversary or by the psychological impact of the importance of the competition. By measuring the nasal resistance before and after physical activity, we have demonstrated that these should not drop in a similar pattern as during athletic performance without mask. The mask hinders the incoming air from freely accessing the nose and causes the highest inspiratory nasal resistance among the variables considered.

The major limitation of this study was that exercise without the mask was not a fencing assault considering the hazard of performing this test; however the physical activity without mask was very similar to the one in a real assault. From the results of this study new mask shapes could be designed.

The fencing masks prevent a condition of optimal nasal breathing; further studies will be needed to test the effectiveness of devices such as nasal dilator strips, to prove their utility in decreasing breathing impairment in athletes.

\section{Acknowledgements}

Special thanks to Lio Bastianini, Technical Manager, CUS Siena, for the recruitment of fencers and for the hospitality extended to us in his gym.

\section{References}

1. Scadding G, Hellings P, Alobid I, Bachert C, Fokkens W, van Wijk $\mathrm{RG}$, et al. Diagnostic tools in Rhinology EAACI position paper. Clin Transl Allergy. 2011;1(1):2.

2. Shelton DM, Eiser NM. Evaluation of active anterior and posterior rhinomanometry in normal subjects. Clin Otolaryngol Allied Sci.1992;17(2):178-82.

3. Bussieres M, Perusse L, Leclerc JE. Effect of regular physical exercise on resting nasal resistance. J Otolaryngol. 2000;29(5):265-9.

4. Fonseca MT, Voegels RL, Pinto KM. Evaluation of nasal volume by acoustic rhinometry before and after physical exercise. Am J Rhinol.2006;20(3):269-73.

5. Bennett WD, Zeman KL, Jarabek AM. Nasal contribution to breathing with exercise: effect of race and gender. J Appl Physiol (1985). 2003;95(2):497-503.

6. Gehring JM, Garlick SR, Wheatley JR, Amis TC. Nasal resistance and flow resistive work of nasal breathing during exercise: effects of a nasal dilator strip. J Appl Physiol (1985). 2000;89(3):1114-22.

7. Wilde AD, Ell SR. Effect on nasal resistance of an external nasal splint and isotonic exercise. BrJ Sports Med.1999;33(2):127-8.

8. Wong LS, Johnson AT. Decrease of resistance to air flow with nasal strips as measured with the airflow perturbation device. Biomed Eng Online. 2004;3(1):38. 